IOS Press

\title{
Development and application of international guidelines for statistical business registers: Discussant's remarks
}

\author{
Michael Colledge \\ Michael Colledge Statistical Consulting Pty Ltd, Canberra, Australia \\ Tel.: +61 437494 030; Fax: +1 613260 6325; E-mail: michael.colledge@ gmail.com
}

\section{Introduction}

A set of papers on the development and application of international guidelines for statistical business registers (SBRs) were presented at the 2017 World Statistics Conference. The aim of my remarks is to summarize the contents of the papers, and to highlight the key messages they conveyed, as the starting point for general discussion by the participants at the session and for subsequent reflection by the authors.

Three of the papers described international/supranational guidelines for statistical business registers (SBRs). The fourth paper by Ms Eileen Capilit and Ms Talam describes an Asian Development Bank (ADB) SBR development program, which has not resulted in separate set of guidelines.

My remarks are based on a review of each paper, including in the first three cases, the corresponding guidelines. I am looking for commonalities, differences, and discrepancies between the papers, and I have included questions and recommendations for the authors. I conclude with some general observations.

\section{Experiences in application of the European Statistical System Business Registers Recommendations Manual by Mr Amerigo Liotti}

The paper outlines the structure and content of the Business Registers Recommendations Manual (here- after referred to as the Manual) and presents the results of a recent survey of Manual users.

The Manual is the first of the supranational/international guidelines on SBRs to have been written. It has a very specific purpose, namely to explain and provide guidance in the application of SBR regulations to European Statistical System (ESS) countries, thereby supporting harmonization of SBRs across these countries. It was last updated in 2010 specifically to take into account the (then) new BR Regulation (EC) $177 / 2008$. This regulation also legislates on exchange of confidential data within the ESS and is the basis on which the EuroGroups Register (EGR) of multinational enterprise groups has been built. Thus, the Manual covers the provision of microdata from ESS countries to Eurostat to source the EGR.

The Manual is well written and comprehensive, containing 24 chapters in all. The chapters are complementary, but designed so that they can be read independently. They are organized in four groups.

- The basics: objectives, units, contents and access (chapters 1-10), including objectives and uses of SBRs, harmonization, maintenance, contents (characteristics), coverage, the legal unit, the enterprise and the local unit, access, and quality policy.

- Unit demography: changes and continuity (chapters 11-16, 21-22), including the general approach to the handling of changes, the enterprise group, an overview of demographic events, and 
demographic changes and continuity rules for the enterprise, the local unit, and the enterprise group.

- Updates and development (chapters 17-20), including the handling of changes to characteristics, of errors, and of large and complex businesses, and the use of administrative sources.

- Guidelines for specific domains (chapters 23-24), including statistical units in agriculture, forestry and fishing, and in the public sector.

In 2016, Eurostat addressed a short questionnaire to the national statistical institutes (NSIs) in ESS countries to collect their views on the present version of the Manual. The survey was voluntary. Although only 15 NSIs replied, the replies were quite consistent and enabled an acceptable analysis.

Key conclusions from the survey are that the Manual:

- is useful and is used; and

- could be improved, for example, by better distinguishing Eurostat's recommendations from county best practices, and by dealing with new issues, including more details about the EGR version and its usage.

The survey provides input to a revised version of the Manual, which is scheduled for 2018 and which will:

- take into account the upcoming Framework Regulation Integrating Business Statistics;

- incorporate recommendations from the on-going Data Quality Programme for the Statistical Business Registers in the ESS;

- contain more examples and best practices; and

- be in a format that can be continually updated.

The revised version will be part of a broader initiative, called the European Business Statistics (EBS) Manual, the aim of which is to introduce topics that are of interest not only to producers of business statistics, but also to end-users wanting to know more about how statistics are produced.

\section{Questions and recommendations}

- Will the revised version of the Manual be realigned to conform with the structure of the international UNECE SBR Guidelines, or will the Manual retain its existing structure?

- What provisions will be made to ensure there is compatibility between the revised version of the Manual and the current UNECE SBR Guidelines and/or the future UN SBR Guidelines?

- The revised version should include references to the current UNECE SBR Guidelines and/or the future UN SBR Guidelines and should highlight where (if at all) the Manual diverges from these guidelines and why. This will help NSOs decide which set of guidelines is most appropriate for their needs.

3. Development and application of African Development Bank Statistical Business Register Guidelines by Mr Vikash Madhow Consultant, Mr Besa Muwele and Mr Abdool Sariff Mungralee

The Guidelines for Building Statistical Business Registers in Africa (hereafter referred to as the AfDB SBR Guidelines, or simply the Guidelines) were developed by the African Development Bank (AfDB) in 2012, with the hard copy version being available in 2014. The Guidelines drew on the Business Registers Recommendations Manual. Thus, there are no substantive discrepancies between the Guidelines and the Manual. However, the Guidelines do include some simplifications to make them more easily readable than the Manual and more suitable for countries that have a limited SBR, or no SBR. For example, the Guidelines specify a one-to-one relationship between a legal entity and an enterprise, whereas the Manual allows a more complex relationship. Although the Guidelines are targeted at African countries they are equally applicable to any country with a limited, or no, SBR.

The paper does not describe the Guidelines per se as they were presented to the 2015 World Statistics Congress. Instead it focuses on development of a generic SBR System that supports SBR operations and that can be readily tailored to the needs of any particular country. Development is based on premise that all countries that follow the AfDB SBR Guidelines should, in principle, be able to make use of the same core SBR system components. An example of an existing (but limited) generic SBR system is the Ficheiro de Unidades Estatísticas (FUE), developed for Portuguese speaking African countries with support from Statistics Portugal.

The paper outlines the generic SBR System design goals, and how they can be achieved, as follows.

- Accuracy: meaning that the SBR provides an accurate depiction of the businesses active in the country at any point, including sufficient information to allow sampling by characteristics of the businesses, and that these characteristics must be accurately recorded. This goal is achieved 
through identification and use of the most appropriate administrative sources and of precedence rules to rank the reliability of each source with respect to each characteristic.

- Low latency: meaning that there is minimum time lag between a business event (such as the birth of a business or a change in its principal activity) happening in the economy and the event being reflected in the SBR. This goal is achieved by frequent, automated updating from the selected sources.

- Persistence: meaning that there is a stable view of the SBR that enables consistent frame extraction and sample selection across surveys at certain points in time, even though the SBR itself is being continuously updated with the latest available information to ensure accuracy and low latency. This goal is achieved through creation of snapshots (frozen versions) of the SBR at regular intervals that form the basis for survey frames.

- Sole source of truth: meaning that the SBR is the only source of business survey frames in the NSO as the use of other sources for individual surveys increases the risk of conflicting information on businesses, and thwarts the goal of harmonization. This goal is achieved by incorporating the survey frame creation and sample selection functions within the SBR System.

The paper outlines the initial prototype generic SBR System developed to support Statistics Mauritius and a subsequent, more flexible version developed for Ethiopian Central Statistical Agency. It indicates some of the systems related problems that were encountered and their means of resolution, including that:

- updating from the principal administrative source must be automated otherwise SBR staff are not able to keep abreast of the updates;

- automated updating must not take too long and must be able to cope with power outages that occur during the updating process; and

- the System must support multiple languages.

The paper also outlines several other (i.e., not systems) problems encountered in the Mauritian context and how they have been addressed.

- The intended primary data source, namely tax data, has not yet been made available by the Mauritius Revenue Authority. To deal with this situation, business registration data from the Register of Companies (ROC) are used as an alternative primary source.
- ROC data do not indicate if a registered business is in active production. To obtain such information, supplementary data from Mauritius Social Security are used.

- The frame for the Survey of Employment and Earnings (SEE) derived from the SBR does not contain some recently formed new businesses. The approach adopted is to supplement the SEE frame with a supplementary frame developed by the SEE.

\section{Questions and recommendations}

- For clarity, the accuracy design goal should be divided into three design goals - comprehensive coverage, comprehensive content and accuracy of content.

- Based on what information is the SEE based supplementary frame maintained?

- Given that supplementary frame is specific to the SEE, does it not violate the sole source of truth design goal?

- Can the SEE based supplementary frame be used as a second primary source, i.e., to update the SBR itself?

\section{Development and application of the SBR Guidelines of the Conference of European Statisticians by Norbert Rainer}

The paper outlines the Guidelines on Statistical Business Registers (hereafter referred to as the UNECE SBR Guidelines or the Guidelines) and then describes the results of a voluntary survey of user countries that was conducted one year after the Guidelines were first published.

The Guidelines were developed by a United Nations Economic Commission for Europe (UNECE) task force over three years, 2012-2015. They were in response to suggestions by two international SBR Working Groups (the Wiesbaden Group and the Joint UNECE/Eurostat/OECD SBR Working Group). As a result of the timing, the authors could draw on the BR Recommendations Manual and on the AfDB SBR Guidelines. Thus, although there are differences between the three sets of guidelines at a detailed level, there are no obvious major discrepancies between them.

In terms of intended audience, the UNECE SBR Guidelines have broadest coverage of the three sets of SBR guidelines described in this session. 
There were 32 responses to questionnaire, which shows a reasonably high level of interest by NSOs. The primary findings were that the guidelines should:

- provide additional guidance in the areas of statistical units, software and IT;

- be supported by seminars, workshops, training courses and technical assistance;

- be adapted/extended to become United Nations (UN) SBR guidelines;

- provide more support for countries with less well developed SBRs.

In response to this and other feedback it has been agreed that the guidelines will be adapted and extended to become UN SBR guidelines.

\section{Questions arising from the paper}

- Will the UN version supersede or complement the UNECE version?

- What relationship will the UN version have to the AfDB SBR Guidelines which are targeted at countries with less developed SBRs?

- The UN version should include references to the revised BR Recommendations Manual and the AfDB SBR Guidelines should highlight where (if at all) it diverges from these guidelines and why. This will help NSOs decide which set of guidelines is most appropriate for their needs.

\section{Establishing Statistical Business Registers in Asia by Ms Eileen Capilit and Ms Irene Talam}

The paper describes the Asian Development Bank (ADB) Project for Improved Information on Small, Medium-sized and Large Enterprises in Bhutan, Cambodia, Lao PDR, Malaysia and Sri Lanka that was initiated in 2013. The Project aims to build on and complement ongoing initiatives of ADB's regional departments by:

- strengthening the link between SBRs and administrative sources of business data;

- ensuring that national statistical systems make full use of the data available from administrative sources; and

- improving coordination among national agencies in the collection and compilation of business data.

The Project includes building each NSO's capacity to develop and operate an SBR by providing a standard guide, training staff, establishing an informal network between NSOs, and developing a generic SBR system. It is being implemented in three phases:
- Phase 1 involved conducting SBR meetings and training sessions for NSOs and stakeholders in the five countries, developing of a Prototype $A D B$ SBR System and launching a version of the System in each country. System development was done in house thus facilitating feedback from all parties during the testing.

- Phase 2 involves tailoring the standard SBR framework and Prototype System to the specific circumstances in each country. This includes training NSO staff to develop additional modules to suit their unique country setting, establishing a legal basis for administrative data access, and institutionalizing the partnerships between the NSO and source agencies.

- Phase 3 extends beyond the Project and aims to establish an Integrated Data Infrastructure that will support a cost-efficient, comprehensive and sustainable SBR system that responds to internal and external client needs.

The System has the facility to store, browse, maintain and retrieve information to identify and describe statistical units. It incorporates the concepts defined in the System of National Accounts (SNA) and the International Standard Industrial Classification (ISIC) of Economic Activities, and it includes concordance tables to facilitate mapping national classification systems to ISIC.

\section{Questions and recommendations}

- Has a set of SBR guidelines been produced by the Project?

- If yes, are they available?

- If no, specifically which set of international guidelines have been recommended to the NSOs?

- Has an operational SBR been established in any of the countries?

- If yes, which countries and with what features?

- The Project should consider using the AfDB based generic SBR System as the latter is further developed and has more functionality.

\section{Concluding remarks}

All the papers give useful insights into design and development of SBRs. They have an entirely common approach regarding the objectives of an SBR. They agree that an SBR is vital core infrastructure for economic statistics and that it should: 
- provide frames that have known coverage and are coherent with one another for all business surveys;

- promote common conceptual standards, including the SNA 2008 and ISIC Rev 4;

- be a source of economic statistics in its own right; and

- link and integrate business microdata.

There are differences in degree of detail provided by the three sets of SBR guidelines but no obvious substantive discrepancies.

The generic SBR systems developed by the AfDB and the ADB have similar goals. The AfDB System has significantly greater functionality.

\section{Recommendations}

- The relationships between the three sets of SBR guidelines should be formalized so that an NSO can readily determine which set of guidelines is most appropriate for its particular circumstances.

- Other international SBR guidelines, including for the Caribbean and the South Pacific Community, should be brought into the discussion.

- Implementation of SBR guidelines would be greatly facilitated by the promotion of a generic SBR system. 